\title{
AN INDIRECT SUFFICIENCY PROOF FOR THE PROBLEM OF LAGRANGE WITH DIFFERENTIAL INEQUALITIES AS ADDED SIDE CONDITIONS
}

BY

\section{LOUIS L. PENNISI}

1. Introduction. The problem to be considered here consists in finding in a class of $\operatorname{arcs} C: y^{i}(x)\left(i=1, \cdots, n ; x^{1} \leqq x \leqq x^{2}\right)$ joining two fixed points and satisfying a set of differential inequalities and equations of the form

$$
\phi^{\beta}(x, y, \dot{y}) \geqq 0, \quad \psi^{\rho}(x, y, \dot{y})=0
$$

that one which minimizes the integral

$$
I(C)=\int_{x^{1}}^{x^{2}} f(x, y, \dot{y}) d x .
$$

Valentine [11](1) has given a brief history of this problem and has derived certain necessary conditions by introducing auxiliary functions $z^{\beta}(x)$ such that $\phi^{\beta}(x, y, \dot{y})=\dot{z}^{\beta^{2}}$. His sufficiency theorems depended on assumptions of normality and a field theory, and also required all except one of the differential inequalities to be satisfied in the strict sense along the minimizing arc $C_{0}$.

Using methods developed by McShane [9] and Hestenes $[3 ; 4 ; 5 ; 6]$ we shall give an indirect proof of a sufficiency theorem. Instead of demanding that all but one of the functions $\phi^{\beta}$ is positive along $C_{0}$ we shall impose the more general restriction (2.5) to be described in the next section.

2. Statement of problem and main theorem. Let $R$ be a region in $(2 n+1)$ dimensional space of points $(x, y, p)=\left(x, y^{1}, \cdots, y^{n}, p^{1}, \cdots, p^{n}\right)$. By an admissible arc $C$ will be meant a set of functions $y^{i}(x)\left(i=1, \cdots, n ; x^{1} \leqq x \leqq x^{2}\right)$ which are absolutely continuous and have integrable square derivatives $\dot{y}^{i}(x)$ such that the point $[x, y(x), \dot{y}(x)]$ is in $R$ for almost all $x$ on $x^{1} x^{2}$. It will be assumed that the functions $f(x, y, p), \phi^{\beta}(x, y, p), \psi^{\rho}(x, y, p)$ are of class $C^{\prime \prime}$ on $R(\beta=1, \cdots, m ; \rho=m+1, \cdots, m+t<n)$. The subset on $R$ on which $\phi^{\beta}(x, y, p) \geqq 0, \psi^{\rho}(x, y, p)=0$ will be denoted by $\mathcal{D}$. We shall say that $C$ lies in $\mathcal{D}$ if the point $[x, y(x), \dot{y}(x)]$ lies in $\mathcal{D}$ for almost all $x$ on $x^{1} x^{2}$.

We shall be concerned with a particular admissible arc $C_{0}: y^{i}=y_{0}^{i}(x)$ of class $C^{\prime}$ which lies in $\mathcal{D}$, satisfies the end conditions

$$
y^{i}\left(x^{s}\right)=y^{i s} \quad\left(i=1, \cdots, n ; x^{1} \leqq x \leqq x^{2} ; s=1,2\right)
$$

and along which the matrix

Received by the editors April 4, 1952.

(1) The numbers in brackets refer to the bibliography at the end of the paper. 


$$
\left\|\begin{array}{c}
\phi_{p^{i}}^{\beta} \\
\psi_{p^{i}}^{\rho}
\end{array}\right\|
$$

has rank $m+t$. Suppose that the interval $x^{1} x^{2}$ can be divided into a finite number of open intervals $A_{\tau}: x_{\tau}<x<x_{\tau+1}(\tau=0,1, \cdots, T)$, where $x_{0}=x^{1}$, $x_{T+1}=x^{2}$, in such a manner that each of the functions $\phi^{\beta}\left[x, y_{0}(x), \dot{y}_{0}(x)\right]$ either vanishes identically on $A_{\tau}$ or is positive everywhere on $A_{\tau}$. Let $\Gamma(x)$ be the set of indices $\beta$ such that $\phi^{\beta}\left[x, y_{0}(x), \dot{y}_{0}(x)\right]=0$. Then $\Gamma(x)$ is independent of $x$ when $x$ is in $A_{\tau}$ and may be denoted by $\Gamma_{\tau}$. Let $A(\beta)$ be the closure of the sum of the intervals $A_{\tau}$ on which $\phi^{\beta}\left[x, y_{0}(x), \dot{y}_{0}(x)\right]=0$.

Consider now a set of continuous functions $\lambda^{0} \geqq 0, \lambda^{\beta}(x), \lambda^{\rho}(x)$ such that if we define

$$
F(x, y, p, \lambda)=\lambda^{0} f(x, y, p)+\lambda^{\beta} \phi^{\beta}(x, y, p)+\lambda^{\rho} \psi^{\rho}(x, y, p),
$$

then the equations

$$
\begin{aligned}
& F_{p^{i}}=\int_{x^{1}}^{x} F_{y^{i}} d x+c^{i}, \\
& \lambda^{\beta}(x) \phi^{\beta}(x, y, \dot{y})=0
\end{aligned}
$$

( $\beta$ not summed)

hold along $C_{0}$ with the multipliers $\lambda(x)$ for some set of constants $c^{i}$. Let $\Delta(x)$ be the set of indices $\beta$ such that $\lambda^{\beta}(x) \neq 0$ and let $B(\beta)$ be the set of points $x$ for which $\lambda^{\beta}(x) \neq 0$. It is evident from (2.4) that $\bar{B}(\beta)$, the closure of $B(\beta)$, is contained in $A(\beta)$ and that $\Delta(x)$ is contained in $\Gamma(x)$. In fact, $\Delta(x)$ is contained in $\Gamma_{\tau}$ for all $x$ in $\bar{A}_{\tau}$. We shall assume that

$$
\Gamma(x)-\Delta(x) \text { contains at most one index. }
$$

We shall make a further restriction on our choice of multipliers. Let $E_{F}(x, y, p, q, \lambda)$ be the Weierstrass $E$-function

$$
E_{F}=F(x, y, q, \lambda)-F(x, y, p, \lambda)-\left(q^{i}-p^{i}\right) F_{p^{i}}(x, y, p, \lambda),
$$

and define $\Phi^{\beta}(x, y, p)$ as

$$
\phi^{\beta}(x, y, p)=\phi^{\beta}(x, y, p) /\left[1+\phi^{\beta^{2}}(x, y, p)\right]^{1 / 2} .
$$

It will be assumed that there is a neighborhood $\mathcal{D}_{1}$ of $C_{0}$ relative to the set $\mathcal{D}$ and a constant $b$ such that $0<b<1$ and the inequality

$$
E_{F}(x, y, p, q, \lambda)-\lambda^{\beta}(x) \phi^{\beta}(x, y, q) \geqq b E_{L}(p, q)-\lambda^{\beta}(x) \Phi^{\beta}(x, y, q)
$$

holds whenever $(x, y, q)$ is in $\mathcal{D}_{1},(x, y, q)$ is in $\mathscr{D}$, and $\phi^{\beta}(x, y, p)=0$ in case $\beta$ is in $\Delta(x)$. Here $L(p)$ is the integrand $\left(1+p^{i} p^{i}\right)^{1 / 2}$ of the length integral and $E_{L}(p, q)$ is the $E$-function

$$
E_{L}(p, q)=L(q)-\left(1+p^{i} q^{i}\right) / L(p)
$$


for this integrand. We shall call the set of functions $\lambda^{0} \geqq 0, \lambda^{\beta}(x), \lambda^{\rho}(x)$ an admissible set of multipliers when the functions are continuous on $x^{1} x^{2}$ and the conditions (2.3), (2.4), (2.5), and (2.6) are satisfied under the conditions described.

Let us now consider a set of functions $\eta^{i}(x)$ which are absolutely continuous and have integrable square derivatives $\dot{\eta}^{i}(x)$ on $x^{1} x^{2}$. If such a set of functions satisfies with the curve $C_{0}$ and an admissible set of multipliers the end conditions

$$
\eta^{i}\left(x^{s}\right)=0,
$$

the differential equations

$$
\phi_{y i \eta}^{\beta} \eta^{i}+\phi_{p i \eta^{\beta}}^{\beta}=0
$$

for almost all $x$ in $B(\beta)$, the differential inequalities

$$
\phi_{y i \eta}^{\beta}+\phi_{p^{i} \dot{\eta}^{i}}^{\boldsymbol{\beta}} \geqq 0
$$

for almost all $x$ in $A(\beta)-B(\beta)$, and the differential equations

$$
\psi_{y i \eta}^{\rho} \eta^{i}+\psi_{p i \eta^{\rho}}^{i}=0
$$

for almost all $x$ on $x^{1} x^{2}$, it will be called an admissible variation. For each admissible variation, the second variation

$$
\begin{aligned}
J_{2}(\eta) & =\int_{x^{1}}^{x^{2}} 2 \omega(x, \eta, \dot{\eta}) d x \\
& =\int_{x^{1}}^{x^{2}}\left(F_{y^{i} y^{\prime} k} \eta^{i} \eta^{k}+2 F_{y^{i} p^{k} \eta^{i} \dot{\eta}^{k}}+F_{p^{i} p^{k} \dot{\eta}^{i} \dot{\eta}^{k}}\right) d x
\end{aligned}
$$

is well defined.

Theorem 2.1. Let $C_{0}$ be an arc of class $C^{\prime}$ which lies in $\mathcal{D}$, satisfies the end conditions (2.1), and along which the matrix (2.2) has rank $m+t$. Suppose an admissible set of multipliers can be found for which $J_{2}(\eta)>0$ for every nonnull admissible variation. Then there is a neighborhood $\mathcal{F}$ of $C_{0}$ in $(x, y)$-space such that the inequality $I(C)>I\left(C_{0}\right)$ holds for every admissible arc $C$ in $\mathcal{F}$ which lies in $\mathcal{D}$, satisfies the end conditions (2.1), and is different from $C_{0}$.

3. The problem with a finite number of variables. In order to be able to draw conclusions from the inequality (2.6) and also to furnish a model for the calculus of variations problem, we find it convenient to discuss first the problem of minimizing a function $f(x)$ of $n$ variables $x^{i}$ in the class of points $x$ satisfying $m$ inequalities $\phi^{\beta}(x) \geqq 0$ and $t$ equations $\psi^{\rho}(x)=0$, when $m+t<n$. For the normal case, which is the only one we consider, our results are more general than those of Karush [7]. 
We suppose that the functions $f(x), \phi^{\beta}(x)$, and $\psi^{\rho}(x)$ are of class $C^{\prime \prime}$ in a neighborhood $R$ of a point $x_{0}$, that $\phi^{\beta}\left(x_{0}\right) \geqq 0, \psi^{\rho}\left(x_{0}\right)=0$ and that the matrix

$$
\left\|\begin{array}{l}
\phi_{x i}^{\beta}\left(x_{0}\right) \\
\psi_{x i}^{p}\left(x_{0}\right)
\end{array}\right\|
$$

has rank $m+t$.

THEOREM 3.1. If $x_{0}$ minimizes $f(x)$ in the class of points $x$ near $x_{0}$ such that $\phi^{\beta}(x) \geqq 0, \psi^{\rho}(x)=0$, then there exist unique multipliers $\mu^{\beta}, \mu^{\rho}$ such that if $F(x, \mu)=f(x)+\mu^{\beta} \phi^{\beta}(x)+\mu^{\alpha} \psi^{\rho}(x)$, then $F_{x^{i}}\left(x_{0}, \mu\right)=0$. Moreover, $\mu^{\beta} \leqq 0$ and $\mu^{\beta} \phi^{\beta}\left(x_{0}\right)=0$ for each $\beta$.

The first sentence of the theorem follows from well known results $[1, \mathrm{p}$. 210 ] since the point $x_{0}$ is a normal point which minimizes $f(x)$ in the class of points $x$ near $x_{0}$ for which $\phi^{\beta}(x)=\phi^{\beta}\left(x_{0}\right), \psi^{\rho}(x)=0$. To prove that $\mu^{\beta} \leqq 0$ we pick functions $\psi^{j}(x)(j=m+t+1, \cdots, n)$ of class $C^{\prime \prime}$ such that the equations

$$
\phi^{\beta}(x)=v^{\beta}+\phi^{\beta}\left(x_{0}\right), \quad \psi^{p}(x)=0, \quad \psi^{j}(x)=\psi^{j}\left(x_{0}\right)+v^{i}
$$

have a nonvanishing functional determinant when $x=x_{0}, v^{\beta}=0, v^{j}=0$. Solutions $x^{i}(v)$ of class $C^{\prime \prime}$ near $v=0$ therefore exist such that $x^{i}(0)=x_{0}^{i}$ and so the function

$$
f[x(v)]=F[x(v), \mu]-\mu^{\beta} v^{\beta}-\mu^{\beta} \phi^{\beta}\left(x_{0}\right)
$$

is minimized by $v=0$ in the class of points $v$ near 0 for which each $v^{\beta} \geqq 0$. Its derivative with respect to $v^{\beta}$, namely $-\mu^{\beta}$, must therefore be non-negative. If $\phi^{\beta}\left(x_{0}\right)>0$ for some $\beta$, then the function (3.3) has a two-sided minimum when regarded as a function of $v^{\beta}$ only and so $\mu^{\beta}=0$. Hence $\mu^{\beta} \phi^{\beta}\left(x_{0}\right)=0$ for each $\beta$.

Let us define $\Gamma$ as the set of all indices $\beta$ such that $\phi^{\beta}\left(x_{0}\right)=0$, and $\Delta$ as the set of all indices $\beta$ such that $\mu^{\beta}<0$. It is clear then that $\Delta$ is a subset of $\Gamma$.

THEOREM 3.2. If $x_{0}$ minimizes $f(x)$ in the class of points $x$ near $x_{0}$ such that $\phi^{\beta}(x) \geqq 0, \psi^{\rho}(x)=0$, then the function $F(x, \mu)$ defined by Theorem 3.1 is such that

$$
F_{x^{i} x^{k}}\left(x_{0}, \mu\right) \pi^{i} \pi^{k} \geqq 0
$$

for every set $\pi^{i}$ such that

$$
\psi_{x^{i}}^{p}\left(x_{0}\right) \pi^{i}=0, \quad \phi_{x i}^{\gamma}\left(x_{0}\right) \pi^{i} \geqq 0, \quad \phi_{x i}^{\delta}\left(x_{0}\right) \pi^{i}=0
$$

for all $\rho$, all $\gamma$ in $\Gamma-\Delta$, and all $\delta$ in $\Delta$.

If $\pi^{i}$ is a set as described in the theorem, we set $v^{\beta}(t)=\phi_{x}^{\beta}\left(x_{0}\right) \pi^{i} t, v^{i}(t)$ $=\psi_{x^{i}}^{i}\left(x_{0}\right) \pi^{i} t$ and observe that the function $f[x\{v(t)\}]$, in which $x(v)$ is the function constructed in the proof of Theorem 3.1, is minimized by $t=0$ in 
the class of non-negative $t$ near $t=0$. The first derivative of this function obviously vanishes when $t=0$ and so its second derivative must be non-negative when $t=0$, or $F_{x^{i} x^{k}}\left(x_{0}, \mu\right) \dot{x}_{0}^{i} \dot{x}_{0}^{k} \geqq 0$, in which $\dot{x}_{0}^{i}=d x^{i}[v(t)] / d t$ when $t=0$. If we differentiate equations (3.3) after replacing $v$ by $v(t)$, we discover that

$$
\begin{gathered}
\phi_{x i}^{\beta}\left(x_{0}\right) \dot{x}_{0}^{i}=\phi_{x_{i}}^{\beta}\left(x_{0}\right) \pi^{i}, \quad \psi_{x i}^{p}\left(x_{0}\right) \dot{x}_{0}^{i}=0=\psi_{x i}^{p}\left(x_{0}\right) \pi^{i}, \\
\psi_{x i}^{j}\left(x_{0}\right) \dot{x}_{0}^{i}=\psi_{x i}^{j}\left(x_{0}\right) \pi^{i} .
\end{gathered}
$$

It follows that $\dot{x}_{0}^{i}=\pi^{i}$ and hence that $F_{x^{i} x^{k}}\left(x_{0}, \mu\right) \pi^{i} \pi^{k} \geqq 0$, as desired.

CoRollary. If $x_{0}$ minimizes $f(x)$ in the class of points $x$ near $x_{0}$ such that $\phi^{\beta}(x) \geqq 0, \psi^{\rho}(x)=0$, and if the set $\Gamma-\Delta$ contains at most one index, $\gamma$, then the function $F(x, \mu)$ defined by Theorem 3.1 is such that $F_{x^{i} x^{k}}\left(x_{0}, \mu\right) \pi^{i} \pi^{k} \geqq 0$ for every set $\pi^{i}$ such that $\psi_{x^{i}}^{\rho}\left(x_{0}\right) \pi^{i}=0, \phi_{x^{i}}^{\delta}\left(x_{0}\right) \pi^{i}=0$ for all $\rho$ and all $\delta$ in $\Delta$.

There is nothing new to prove unless $\pi^{i}$ is a set satisfying the conditions of the corollary for which $\phi_{x}^{\gamma}\left(x_{0}\right) \pi^{i}<0$. In this case we define $\bar{\pi}^{i}=-\pi^{i}$ and have a set $\bar{\pi}^{i}$ satisfying the conditions of Theorem 3.2. Since the quadratic form is unaltered when $\pi^{i}$ is replaced by $\bar{\pi}^{i}$, we see that the corollary is true.

THEOREM 3.3. Suppose there exist multipliers $\mu^{0} \geqq 0, \mu^{\beta} \leqq 0, \mu^{\rho}$ such that if $F(x, \mu)=\mu^{0} f(x)+\mu^{\beta} \phi^{\beta}(x)+\mu^{\rho} \psi^{\rho}(x)$, then $F_{x^{i}}\left(x_{0}, \mu\right)=0, \mu^{\beta} \phi^{\beta}\left(x_{0}\right)=0$ for each $\beta$. If the quadratic form $F_{x^{i} x^{k}}\left(x_{0}, \mu\right) \pi^{i} \pi^{k}>0$ for all nonnull $\pi^{i}$ such that $\psi_{x^{i}}\left(x_{0}\right) \pi^{i}$ $=0, \phi_{x}^{\gamma}\left(x_{0}\right) \pi^{i} \geqq 0, \phi_{x}^{\delta}\left(x_{0}\right) \pi^{i}=0$ for all $\rho$, all $\gamma$ such that $\mu^{\gamma}=\phi^{\gamma}\left(x_{0}\right)=0$, and all $\delta$ such that $\mu^{\delta}<0$, then there is a neighborhood $\mathcal{F}$ of $x_{0}$ such that $f(x)>f\left(x_{0}\right)$ if $x$ is any point in $\mathcal{F}$ different from $x_{0}$ for which $\phi^{\beta}(x) \geqq 0, \psi^{\rho}(x)=0$.

If this theorem were false, there would be a sequence of points $x_{r} \neq x_{0}$ converging to $x_{0}$ such that $\phi^{\beta}\left(x_{r}\right) \geqq 0, \psi^{\rho}\left(x_{r}\right)=0, f\left(x_{r}\right) \leqq f\left(x_{0}\right)$. Define $k_{r}$ as the positive square root of $\left|x_{r}-x_{0}\right|^{2}-\mu^{\beta} \phi^{\beta}\left(x_{r}\right)$. Then $k_{r}$ converges to zero. If we define $\pi_{r}^{i}=\left(x_{r}^{i}-x_{0}^{i}\right) / k_{r}$, then $\left|\pi_{r}\right| \leqq 1$ since $-\mu^{\beta} \phi^{\beta}\left(x_{r}\right) \geqq 0$. By passing to a subsequence we may therefore suppose that $\pi_{r}^{i}$ converges to a limit $\pi_{0}^{i}$. Since $\mu^{0} \geqq 0$, we have that

$$
0 \geqq k_{r}^{-2}\left[F\left(x_{r}, \mu\right)-F\left(x_{0}, \mu\right)-\mu^{\beta} \phi^{\beta}\left(x_{r}\right)\right] .
$$

Expanding the right-hand side of this inequality by Taylor's theorem, making use of the fact that $F_{x_{i}}\left(x_{0}, \mu\right)=0$, and letting $r$ approach $\infty$, we find that

$$
0 \geqq \frac{1}{2} F_{x i x i}\left(x_{0}, \mu\right) \pi_{0}^{i} \pi_{0}^{j}+\lim \sup \left[-k_{r}^{-2} \mu^{\beta} \phi^{\beta}\left(x_{r}\right)\right] .
$$

Moreover, we see from Taylor's theorem and the relations

$$
-\frac{k_{r}}{u^{\delta}}>\frac{\phi^{\delta}\left(x_{r}\right)}{k_{r}} \geqq 0, \quad \frac{\phi^{\gamma}\left(x_{r}\right)}{k_{r}} \geqq 0, \quad \frac{\psi^{\rho}\left(x_{r}\right)}{k_{r}}=0,
$$


that $\phi_{x^{i}}^{\delta}\left(x_{0}\right) \pi_{0}^{s}=0$ if $u^{\delta}<0, \phi_{x}^{\gamma}\left(x_{0}\right) \pi_{0}^{\gamma} \geqq 0$ if $\phi^{\gamma}\left(x_{0}\right)=0$, and $\psi_{x^{i}}^{p}\left(x_{0}\right) \pi_{0}^{i}=0$. If the set $\pi_{0}^{s}$ were nonnull, it would then follow from the hypothesis of Theorem 3.3 that $F_{x_{i x} i}\left(x_{0}\right) \pi_{0}^{i} \pi_{0}^{j}>0$ and so (3.4) could not hold since lim sup [- $\left.-k_{r}^{-2} \mu^{\beta} \phi^{\beta}\left(x_{r}\right)\right]$ $\geqq 0$. Hence $\pi_{0}^{i}=0$. It then follows from (3.4) that

$$
\lim k_{r}^{-2} \mu^{\beta} \phi^{\beta}\left(x_{r}\right)=0 .
$$

Since we have from the definition of $k_{r}$ that $1=\left|\pi_{r}\right|^{2}-k_{r}^{-2} \mu^{\beta} \phi^{\beta}\left(x_{r}\right)$, and since $\pi_{0}^{i}=0$, we see that (3.5) cannot hold. We infer the truth of the theorem from this contradiction.

4. Some consequences of the hypotheses. For a fixed $x$ on $x^{1} x^{2}$ it follows from our hypotheses (2.4) and (2.6) that the function of $q$,

$$
E_{F}\left(x, y_{0}, \dot{y}_{0}, q, \lambda\right)-b E_{L}\left(\dot{y}_{0}, q\right)-\lambda^{\beta}(x) \phi^{\beta}\left(x, y_{0}, q\right)+b \lambda^{\beta}(x) \Phi^{\beta}\left(x, y_{0}, q\right),
$$

is minimized by $q^{i}=\dot{y}_{0}^{i}$ in the class of $q^{i}$ near $\dot{y}_{0}^{i}$ for which $\phi^{\beta}\left(x, y_{0}, q\right) \geqq 0$, $\psi^{\rho}\left(x, y_{0}, q\right)=0$. From Theorem 3.1 we conclude that there exist multipliers $\mu^{\beta}, \mu^{\rho}$ such that

$$
\left[\mu^{\beta}-(1-b) \lambda^{\beta}\right] \phi_{p^{i}}^{\beta}+\mu^{\rho} \psi_{p^{i}}^{p}=0
$$

when $y^{i}=y_{0}^{i}(x), p^{i}=\dot{y}_{0}^{i}(x)$. Since the matrix (2.2) has rank $m+t$, it follows that $\mu^{\beta}=(1-b) \lambda^{\beta}, \mu^{\rho}=0$ and since $0<b<1$, we also conclude from Theorem 3.1 that $\lambda^{\beta} \leqq 0$. From Theorem 3.3 we have that

$$
\frac{\partial^{2}}{\partial q^{i} \partial q^{k}}\left[E_{F}\left(x, y_{0}, \dot{y}_{0}, q, \lambda\right)-b E_{L}\left(\dot{y}_{0}, q\right)\right] \pi^{i} \pi^{k} \geqq 0
$$

when $q^{i}=\dot{y}_{0}^{i}(x)$ for all $\pi^{i}$ such that $\psi_{p}^{p} ; \pi^{i}=0, \phi_{p}^{\gamma} ; \pi^{i} \geqq 0, \phi_{p}^{\delta} ; \pi^{i}=0$ for all $\rho$, all $\gamma$ in $\Gamma(x)-\Delta(x)$ and all $\delta$ in $\Delta(x)$. Since (2.5) holds we have the following theorem.

THEOREM 4.1. If the multipliers satisfy (2.4) and (2.6), then

$$
\lambda^{\beta}(x) \leqq 0 .
$$

If they also satisfy (2.5), then $F_{p^{i} p^{k}} \pi^{i} \pi^{k} \geqq b L_{p^{i} p^{k}} \pi^{i} \pi^{k}$ holds for every set $\pi^{i}$ for which $\psi_{p}^{\rho} ; \pi^{i}=\phi_{p}^{\delta} ; \pi^{i}=0$ for all $\rho$ and all $\delta$ in the set $\Delta(x)$ for which $\lambda^{\delta}(x)<0$.

Corollary 1. If the multipliers satisfy (2.4), (2.5), and (2.6), then the matrix

$$
\left\|\begin{array}{lll}
F_{p^{i} p^{k}} & \phi_{p^{i}}^{\gamma} & \psi_{p^{i}}^{p} \\
\phi_{p^{k}}^{\gamma} & 0 & 0 \\
\psi_{p^{k}}^{p} & 0 & 0
\end{array} \mid\right\|,
$$

in which $\gamma$ ranges over the set $\Gamma_{\tau}$, is nonsingular when $x$ is in $\bar{A}_{\tau}, y^{i}=y_{0}^{i}(x)$, $p^{i}=\dot{y}_{0}^{\prime}(x)$. 
This result is an obvious corollary of Theorem 4.1 when it is remarked that $\Delta(x)$ is a subset of $\Gamma_{\tau}$ when $x$ is in $\bar{A}_{\tau}$.

The following differentiability theorem is an immediate consequence of the preceding corollary.

Corollary 2. When the multipliers are admissible, they and the functions $\dot{y}_{0}^{\prime}(x)$ are of class $C^{\prime}$ on each interval $\bar{A}_{\tau}$.

COROLlary 3. There exists a constant $\theta$ such that if we define

$$
\phi_{0}^{\beta}(x, y, p)=\lambda^{\beta}(x) \phi^{\beta}(x, y, p),
$$

for each $\beta$, and

$$
H(x, y, p)=\theta\left[\psi^{p}(x, y, p) \psi^{\rho}(x, y, p)+\phi_{0}^{\beta}(x, y, p) \phi_{0}^{\beta}(x, y, p)\right],
$$

then the quadratic form $\left(F_{p^{i} p^{k}}+H_{p^{i} p^{k}}\right) \pi^{i} \pi^{k}>0$ for all nonnull vectors $\pi^{i}$ and each $x$ on $x^{1} x^{2}$.

This is a consequence of a known result $\left[10\right.$, p. 679] since $C_{0}$ satisfies the differential equations $\psi^{p}(x, y, \dot{y})=0, \phi_{0}^{\beta}(x, y, \dot{y})=0$ and is such that $F_{p^{i} p_{k}} \pi^{i} \pi^{k}$ $\geqq 0$ for all nonnull vectors $\pi^{i}$ such that $\psi_{p}^{p} ; \pi^{i}=0, \phi_{0 p}^{\beta} ; \pi^{i}=0$.

Lemma 4.1. There exist functions $\psi^{i}(x, y, p)(j=m+t+1, \cdots, n)$ of class $C^{\prime \prime}$ near $C_{0}$ such that the determinant

$$
\left|\begin{array}{l}
\phi_{p^{i}}^{\beta} \\
\psi_{p^{i}}^{p} \\
\psi_{p^{i}}^{j}
\end{array}\right|
$$

does not vanish on $C_{0}$.

This lemma is a well known consequence of the fact that the matrix (2.2) has rank $m+t$ along $C_{0}[1$, pp. 224-226].

5. The equivalence of (2.6) to $\mathrm{II}_{N}$ and condition $\mathrm{III}^{\prime}$. We define the class $\mathcal{N}$ as the collection of all sets $N$ of points $(x, y, p, \nu)$ such that there exists a neighborhood $R_{1}$ of the points $\left(x, y_{0}, \dot{y}_{0}\right)$ on $C_{0}$ and a positive constant $a$ for which $(x, y, p, \nu)$ is in $N$ if and only if $(x, y, p)$ is in $R_{1}, \nu^{0}=\lambda^{0},\left|\nu^{\rho}-\lambda^{\rho}(x)\right|$ $\leqq a,\left|\nu^{\beta}-\lambda^{\beta}(x)\right| \leqq-a \lambda^{\beta}(x)$ if $\beta$ is not in $\Gamma(x)-\Delta(x),-a \leqq \nu^{\gamma} \leqq 0$ if $\gamma$ is in $\Gamma(x)-\Delta(x)$. We shall say that the arc $C_{0}$ with the multipliers $\lambda^{0}, \lambda^{\beta}(x), \lambda^{\rho}(x)$ satisfies the condition $\mathrm{II}_{N}$ if there is a set $N$ in $\mathcal{N}$ such that

$$
E_{F}(x, y, p, q, \nu)-\nu^{\beta} \phi^{\beta}(x, y, q) \geqq 0
$$

for all sets $(x, y, p, q, \nu)$ such that $(x, y, p, \nu)$ is in $N,(x, y, p)$ and $(x, y, q)$ are in $\mathcal{D}$ and $\phi^{\beta}(x, y, p)=0$ if $\beta$ is in $\Delta(x)$.

We shall say that the arc $C_{0}$ with the multipliers $\lambda^{0}, \lambda^{\beta}(x), \lambda^{\rho}(x)$ satisfies the condition III' in case $F_{p^{i} p^{k}}\left[x, y_{0}(x), \dot{y}_{0}(x), \lambda(x)\right] \pi^{i} \pi^{k}>0$ for every nonnull 
vector $\pi^{i}$ such that $\psi_{p^{i}}^{p}\left[x, y_{0}(x), \dot{y}_{0}(x)\right] \pi^{i}=\phi_{p^{i}}^{\delta}\left[x, y_{0}(x), \dot{y}_{0}(x)\right] \pi^{i}=0$ for all $\rho$ and all $\delta$ in $\Delta(x)$.

TheOREM 5.1. If the arc $C_{0}$ with the multipliers $\lambda^{0}, \lambda^{\beta}(x), \lambda^{\rho}(x)$ satisfies equations (2.4), then it satisfies the condition $\mathrm{II}_{N}$ and satisfies the condition $\mathrm{III}^{\prime}$ if and only if there is a constant $b$ such that $0<b<1$ and a neighborhood $\mathcal{D}_{1}$ of $C_{0}$ relative to the set $\mathcal{D}$ such that the inequality (2.6) holds whenever $(x, y, p)$ is in $\mathcal{D}_{1},(x, y, q)$ is in $\mathcal{D}$, and $\phi^{\beta}(x, y, p)=0$ if $\beta$ is in $\Delta(x)$.

The proof of this theorem will follow from a series of lemmas which we now proceed to prove.

Lemma 5.1. If the arc $C_{0}$ with the multipliers $\lambda^{0}, \lambda^{\beta}(x), \lambda^{\rho}(x)$ satisfies the condition $\mathrm{II}_{N}$ and equations (2.4), then $\lambda^{\beta}(x) \leqq 0$.

This follows directly from Theorem 3.1 when we observe that

$$
E_{F}\left(x, y_{0}, \dot{y}_{0}, q, \lambda\right)-\lambda^{\beta}(x) \phi^{\beta}\left(x, y_{0}, q\right) \geqq 0
$$

for any $q^{i}$ such that $\phi^{\beta}\left(x, y_{0}, q\right) \geqq 0, \psi^{p}\left(x, y_{0}, q\right)=0$.

LeMma 5.2. If there is a positive constant $b$ such that the inequality (2.6) holds for all $(x, y, p)$ in a neighborhood $\mathcal{D}_{1}$ of $C_{0}$ relative to $\mathcal{D}$ for which $\phi^{\beta}(x, y, p)$ $=0$ if $\beta$ is in $\Delta(x)$ and all $(x, y, q)$ in $\mathcal{D}$, and if $C_{0}$ with the multipliers $\lambda^{0}, \lambda^{\beta}(x)$, $\lambda \rho(x)$ satisfies the condition $\mathrm{II}_{N}$, then the constant $b$ may be required to be less than one.

If $b$ is not less than one, it follows from Lemma 5.1 that if $b^{\prime}<1$,

$$
b\left[E_{L}(p, q)-\lambda^{\beta}(x) \phi^{\beta}(x, y, q)\right] \geqq b^{\prime}\left[E_{L}(p, q)-\lambda^{\beta}(x) \phi^{\beta}(x, y, q)\right]
$$

for all $(x, y, q)$ in $\mathcal{D}$. Hence the lemma is true.

LEMmA 5.3. If the arc $C_{0}$ with the multipliers $\lambda^{0} \geqq 0, \lambda^{\beta}(x) \leqq 0, \lambda^{\rho}(x)$ satisfies the condition III' and satisfies (2.4), there exists a positive number $b$ and a neighborhood $\mathcal{D}_{0}$ of $C_{0}$ relative to $\mathcal{D}$ such that the inequality (2.6) holds for all $(x, y, p)$ in $\mathcal{D}_{0}$ such that $\phi^{\beta}(x, y, p)=0$ if $\beta$ is in $\Delta(x)$ and all $(x, y, q)$ in $\mathcal{D}_{0}$. 3.3.

The lemma may be proved by minor adaptations of the proof of Theorem

We can easily infer from the identity

$$
\begin{aligned}
E_{F}(x, y, p, q, \lambda)- & \lambda^{\beta}(x) \phi^{\beta}(x, y, q)=E_{F}(x, y, p, q, \nu) \\
& -\nu^{\beta} \phi^{\beta}(x, y, q)+\left(\lambda^{\rho}-\nu^{\rho}\right) E_{\psi^{\rho}}+\left(\lambda^{\beta}-\nu^{\beta}\right)\left(E_{\phi^{\beta}}-\phi^{\beta}\right)
\end{aligned}
$$

and the definition of the class $\mathcal{N}$ that the following lemma is true.

Lemma 5.4. If the arc $C_{0}$ with the multipliers $\lambda^{0}, \lambda^{\beta}(x), \lambda^{\rho}(x)$ satisfies the condition $\mathrm{II}_{N}$, there is a neighborhood $\mathcal{D}_{1}$ of $C_{0}$ relative to the set $\mathcal{D}$ and a positive constant a such that 


$$
E_{F}(x, y, p, q, \lambda)-\lambda^{\beta}(x) \phi^{\beta}(x, y, q) \geqq a\left|E_{\downarrow} \rho(x, y, p, q)\right|
$$

whenever $(x, y, p)$ is in $\mathcal{D}_{1}, \phi^{\beta}(x, y, p)=0$ if $\beta$ is in $\Delta(x)$ and $(x, y, q)$ is in $D$. Moreover, the set $D_{1}$ can be chosen so that for each closed subset $M_{\gamma}$ of $B(\gamma)$ there is a positive constant $a_{\gamma}$ such that

$$
\begin{aligned}
E_{F}(x, y, p, q, \lambda)- & \lambda^{\beta}(x) \phi^{\beta}(x, y, q) \\
& \geqq a_{\gamma}\left|E_{\phi}^{\gamma}(x, y, p, q)-\phi^{\gamma}(x, y, q)\right| \quad(\gamma \text { not summed })
\end{aligned}
$$

whenever $(x, y, p)$ is in $\mathcal{D}_{1}, \phi^{\beta}(x, y, p)=0$ if $\beta$ is in $\Delta(x)$, and $(x, y, q)$ is in $\mathcal{D}$, and $x$ is in $M_{\gamma}$.

The proof of Theorem 5.1 may now be constructed by modifying the method used by Hestenes to prove a similar theorem [3, Theorem 4.3]. If a constant $b$ and a neighborhood $\mathcal{D}_{1}$ having the properties described in Theorem 5.1 cannot be found, it follows from Lemmas 5.2 and 5.3 that there exists a sequence $\left(x_{k}, y_{k}, p_{k}, q_{k}\right)$ such that $\left(x_{k}, y_{k}, p_{k}\right)$ is in $\mathcal{D},\left(x_{k}, y_{k}, q_{k}\right)$ is in $\mathcal{D}-\mathcal{D}_{0}$, $\phi^{\beta}\left(x_{k}, y_{k}, p_{k}\right)=0$ if $\beta$ is in $\Delta\left(x_{k}\right)$,

$$
\begin{aligned}
& E_{F}\left[x_{k}, y_{k}, p_{k}, q_{k}, \lambda\left(x_{k}\right)\right]-\lambda^{\beta}\left(x_{k}\right) \phi^{\beta}\left(x_{k}, y_{k}, q_{k}\right) \\
& \leqq k^{-1}\left[E_{L}\left(p_{k}, q_{k}\right)-\lambda^{\beta}\left(x_{k}\right) \Phi^{\beta}\left(x_{k}, y_{k}, q_{k}\right)\right],
\end{aligned}
$$

and for which $\left(x_{k}, y_{k}, p_{k}\right)$ converges to a point $\left(x_{0}, y_{0}, \dot{y}_{0}\right)$ on $C_{0}$. Since $\lambda^{\beta}(x)$ is continuous, we may suppose that

if $\beta$ is in $\Delta\left(x_{0}\right)$.

$$
\phi^{\beta}\left(x_{k}, y_{k}, p_{k}\right)=0
$$

CASE I. $q_{k}$ has a finite accumulation point $q_{0}$. Then $q_{0}^{i} \neq \dot{y}_{0}^{i}$ for all $i$ since $\left(x_{0}, y_{0}, \dot{y}_{0}\right)$ is on $C_{0}$ and $\left(x_{0}, y_{0}, q_{0}\right)$ is not in $\mathcal{D}_{0}$. It follows from (2.7) that

$$
E_{L}(p, q) \leqq 2 L(q) \text {, }
$$

and since $\Phi^{\beta}(x, y, q) \leqq 1$, it follows from (5.5) and the condition $\mathrm{II}_{N}$ that

$$
\lim \left\{E_{F}\left[\left(x_{k}, y_{k}, p_{k}, q_{k}, \lambda\left(x_{k}\right)\right]-\lambda^{\beta}\left(x_{k}\right) \phi^{\beta}\left(x_{k}, y_{k}, q_{k}\right)\right\}=0 .\right.
$$

Suppose for the moment that the set $\Gamma\left(x_{0}\right)-\Delta\left(x_{0}\right)$ is void. By Lemma 4.1 the equations

$\phi^{\delta}(x, y, r)=0$ if $\delta$ is in $\Delta\left(x_{0}\right), \quad \phi^{\alpha}(x, y, r)=\phi^{\alpha}(x, y, p)+v^{\alpha}$

if $\alpha$ is not in $\Gamma\left(x_{0}\right), \psi^{\rho}(x, y, r)=0, \psi^{j}(x, y, r)=\psi^{j}(x, y, p)+v^{j}$

have solutions $r^{i}(x, y, p, v)$ of class $C^{\prime}$ near $\left(x_{0}, y_{0}, \dot{y}_{0}, 0\right)$ such that $r^{i}\left(x_{0}, y_{0}, \dot{y}_{0}, 0\right)=\dot{y}_{0}^{i},(x, y, r)$ is in $\mathcal{D}$, and $\phi^{\delta}(x, y, r)=0$ if $\delta$ is in $\Delta(x)$. If we set $r_{k}^{i}(v)=r^{i}\left(x_{k}, y_{k}, p_{k}, v\right)$, it follows from the condition $\mathrm{II}_{N}$ that for $|v|$ sufficiently small,

$$
\lim \inf \left\{E_{F}\left[x_{k}, y_{k}, r_{k}(v), q_{k}, \lambda\left(x_{k}\right)\right]-\lambda^{\beta}\left(x_{k}\right) \phi^{\beta}\left(x_{k}, y_{k}, q_{k}\right)\right\} \geqq 0,
$$


and hence from (5.7)

$\lim \inf \left\{E_{F}\left[x_{k}, y_{k}, r_{k}(v), q_{k}, \lambda\left(x_{k}\right)\right]-E_{F}\left[x_{k}, y_{k}, p_{k}, q_{k}, \lambda\left(x_{k}\right)\right]\right\} \geqq 0$,

$$
\begin{aligned}
\left.F\left[x_{0}, y_{0}, \dot{y}_{0}, \lambda\left(x_{0}\right)\right]-\dot{y}_{0}^{i} F_{p^{i}}\left[x_{0}, y_{0}, \dot{y}_{0}, \lambda\right)\left(x_{0}\right)\right] \\
-F\left[x_{0}, y_{0}, r_{0}(v), \lambda\left(x_{0}\right)\right]+r_{0}^{i}(v) F_{p^{i}}\left[x_{0}, y_{0}, r_{0}(v), \lambda\left(x_{0}\right)\right] \\
-q_{0}^{i}\left\{F_{p^{i}}\left[x_{0}, y_{0}, r_{0}(v), \lambda\left(x_{0}\right)\right]-F_{p^{i}}\left[x_{0}, y_{0}, \dot{y}_{0}, \lambda\left(x_{0}\right)\right]\right\}=0 .
\end{aligned}
$$

Define $v^{\alpha}(e)=\phi^{\alpha}\left[x_{0}, y_{0}, \dot{y}_{0}+e\left(q_{0}-\dot{y}_{0}\right)\right]-\phi^{\alpha}\left(x_{0}, y_{0}, \dot{y}_{0}\right)$ if $\alpha$ is not in $\Gamma\left(x_{0}\right)$, $v^{i}(e)=\psi^{j}\left[x_{0}, y_{0}, \dot{y}_{0}+e\left(q_{0}-\dot{y}_{0}\right)\right]-\psi^{i}\left(x_{0}, y_{0}, \dot{y}_{0}\right), r^{i}(e)=r_{0}^{i} v(e)$,

$$
\begin{aligned}
Q(e)= & F\left[x_{0}, y_{0}, \dot{y}_{0}, \lambda\left(x_{0}\right)\right]-\dot{y}_{0}^{i} F_{p^{i}}\left[x_{0}, y_{0}, \dot{y}_{0}, \lambda\left(x_{0}\right)\right] \\
& -F\left[x_{0}, y_{0}, r(e), \lambda\left(x_{0}\right)\right]+r^{i}(e) F_{p^{i}}\left[x_{0}, y_{0}, r(e), \lambda\left(x_{0}\right)\right] \\
& -q_{0}^{i}\left\{F_{p^{i}}\left[x_{0}, y_{0}, r(e), \lambda\left(x_{0}\right)\right]-F_{p^{i}}\left[x_{0}, y_{0}, \dot{y}_{0}, \lambda\left(x_{0}\right)\right]\right\} .
\end{aligned}
$$

By (5.9) and the fact that $r^{i}(0)=\dot{y}_{0}^{*}$ we see that $Q(e) \geqq Q(0)=0$ for all sufficiently small $e$. Hence $Q^{\prime}(0)=0$, or

$$
\left(\dot{y}_{0}^{i}-q_{0}^{i}\right) F_{p^{i} p^{k}}\left[x_{0}, y_{0}, \dot{y}_{0}, \lambda\left(x_{0}\right)\right] \dot{r}^{i}(0)=0 .
$$

Now it follows from (5.3) that $\lim \left|E_{\psi \rho}\left(x_{k}, y_{k}, p_{k}, . q_{k}\right)\right|=0$. Since $\psi^{\rho}\left(x_{k}, y_{k}, p_{k}\right)=\psi^{\rho}\left(x_{k}, y_{k}, q_{k}\right)=0$, it follows that

$$
\left(q_{0}^{i}-\dot{y}_{0}^{i}\right) \psi_{p^{i}}^{p}\left(x_{0}, y_{0}, \dot{y}_{0}\right)=0 .
$$

Similarly, it follows from (5.4) and the fact that $\phi^{\delta}\left(x_{k}, y_{k}, p_{k}\right)=0$ if $\delta$ is in $\Delta\left(x_{0}\right)$ that

$$
\left(q_{0}^{i}-\dot{y}_{0}^{i}\right) \phi_{p^{i}}^{\delta}\left(x_{0}, y_{0}, \dot{y}_{0}\right)=0
$$

if $\delta$ is in $\Delta\left(x_{0}\right)$. If we differentiate the equations satisfied by $r(e)$ and set $e=0$, we find that $\dot{r}^{i}(0)=q_{0}^{i}-\dot{y}_{0}^{t}$ and hence

$$
F_{p^{i} p^{k}}\left[x_{0}, y_{0}, \dot{y}_{0}, \lambda\left(x_{0}\right)\right] \dot{r}^{i}(0) \dot{r}^{k}(0)=0
$$

although the numbers $\dot{r}^{i}(0)$ do not all vanish and satisfy

$$
\psi_{p i}^{p}\left(x_{0}, y_{0}, \dot{y}_{0}\right) \dot{r}^{i}(0)=\phi_{p^{i}}^{\delta}\left(x_{0}, y_{0}, \dot{y}_{0}\right) \dot{r}^{i}(0)=0
$$

for all $\rho$ and all $\delta$ in $\Delta\left(x_{0}\right)$, and this is a contradiction of condition III'.

If the set $\Gamma\left(x_{0}\right)-\Delta\left(x_{0}\right)$ is not void, it contains a unique index $\gamma$. We show first that $\phi_{p}^{\gamma}\left(x_{0}, y_{0}, \dot{y}_{0}\right)\left(q_{0}^{i}-\dot{y}_{0}^{i}\right) \geqq 0$. We observe that if we set $\nu^{\rho}=\lambda^{\rho}, \nu^{\beta}=\lambda^{\beta}$ when $\beta \neq \gamma$, it follows from the identity (5.2) and the condition $\mathrm{II}_{N}$ that if $\nu^{\gamma}=-a$, then the point $\left(x_{k}, y_{k}, p_{k}, \nu\right)$ is in $N$ for $k$ sufficiently large, and

$$
\begin{aligned}
& E_{F}\left[x_{k}, y_{k}, p_{k}, q_{k}, \lambda\left(x_{k}\right)\right]-\lambda^{\beta}\left(x_{k}\right) \phi^{\beta}\left(x_{k}, y_{k}, q_{k}\right) \\
& \quad \geqq-\left[a+\lambda^{\gamma}\left(x_{k}\right)\right]\left[\phi^{\gamma}\left(x_{k}, y_{k}, p_{k}\right)+\left(q_{k}^{i}-p_{k}^{i}\right) \phi_{p^{i}}^{\gamma}\left(x_{k}, y_{k}, p_{k}\right)\right] .
\end{aligned}
$$


Since (5.7) holds and $\lambda^{\gamma}\left(x_{0}\right)=\phi^{\gamma}\left(x_{0}, y_{0}, \dot{y}_{0}\right)=0$, we find that

$$
0 \geqq-a\left(q_{0}^{i}-\dot{y}_{0}^{i}\right) \phi_{p^{i}}^{\gamma}\left(x_{0}, y_{0}, \dot{y}_{0}\right) \text {. }
$$

Since $a>0$, we see that $\phi_{p^{i}}^{\gamma}\left(x_{0}, y_{0}, \dot{y}_{0}\right)\left(q_{0}^{i}-\dot{y}_{0}^{i}\right) \geqq 0$.

Suppose $\phi_{p}^{\gamma}\left(x_{0}, y_{0}, \dot{y}_{0}\right)\left(q_{0}^{i}-\dot{y}_{0}^{i}\right)=0$. Then we include the equation $\phi^{\gamma}(x, y, r)=0$ with the equations (5.8) and the analysis in the preceding paragraphs is unaltered since $\phi_{p}^{\gamma}\left(x_{0}, y_{0}, \dot{y}_{0}\right) \dot{r}^{i}(0)=0$. If $\phi_{p}^{\gamma}\left(x_{0}, y_{0}, \dot{y}_{0}\right)\left(q_{0}^{i}-\dot{y}_{0}^{i}\right)>0$, we include with the equations (5.8) the equation $\phi^{\gamma}(x, y, r)=v^{\gamma}$. Then the solutions $r^{i}(x, y, p, v)$ will be such that $(x, y, r)$ will be in $\mathcal{D}$ if $v^{\gamma} \geqq 0$. We set $v^{\gamma}=\phi^{\gamma}\left[x_{0}, y_{0}, \dot{y}_{0}+e\left(q_{0}-\dot{y}_{0}\right)\right]$, the $v^{\alpha}$ and $v^{i}$ being defined as before. Then $v^{\gamma}(e) \geqq 0$ if $e$ is positive and sufficiently small and so $Q(e) \geqq Q(0)$ for all nonnegative small $e$. Hence $Q^{\prime}(0) \geqq 0$, or $\left(\dot{y}_{0}^{i}-q_{0}^{i}\right) F_{p^{i} p^{k}}\left[x_{0}, y_{0}, \dot{y}_{0}, \lambda\left(x_{0}\right)\right] \dot{r}^{i}(0) \geqq 0$. It follows just as above from (5.11) and (5.12) that $\dot{r}^{i}(0)=q_{0}^{i}-\dot{y}_{0}^{i}$ and that the last inequality cannot hold when $C_{0}$ satisfies condition III'.

This disposes of the case in which $q_{k}$ has a finite accumulation point. Next consider:

CASE II. $L\left(q_{k}\right) \rightarrow+\infty$. Then the sequences $q_{k}^{i} / L\left(q_{k}\right)$ converge to limits $\pi^{i}$ and $\pi^{i} \pi^{i}=1$. It follows from (5.5) and (5.6) that

(5.14) $\lim \left\{E_{F}\left[x_{k}, y_{k}, p_{k}, q_{k}, \lambda\left(x_{k}\right)\right]-\lambda^{\beta}\left(x_{k}\right) \phi^{\beta}\left(x_{k}, y_{k}, q_{k}\right)\right\} / L\left(q_{k}\right)=0$.

If the set $\Gamma\left(x_{0}\right)-\Delta\left(x_{0}\right)$ is void, we define the functions $r_{k}^{4}(v)$ as in the bounded case. Instead of (5.9) we now get

$$
-\pi^{i}\left\{F_{p^{i}}\left[x_{0}, y_{0}, r_{0}(v), \lambda\left(x_{0}\right)-F_{p^{i}}\left[x_{0}, y_{0}, \dot{y}_{0}, \lambda\left(x_{0}\right)\right]\right\} \geqq 0 .\right.
$$

We define $v^{\alpha}(e)=\phi^{\alpha}\left(x_{0}, y_{0}, \dot{y}_{0}+e \pi\right)-\phi^{\alpha}\left(x_{0}, y_{0}, \dot{y}_{0}\right), v^{j}(e)=\psi^{j}\left(x_{0}, y_{0}, \dot{y}+e \pi\right)$ $-\psi^{j}\left(x_{0}, y_{0}, \dot{y}_{0}\right), r^{i}(e)=r_{0}^{i}[v(e)], Q(e)=-\pi^{i}\left\{F_{p^{i}}\left[x_{0}, y_{0}, r(e), \lambda\left(x_{0}\right)\right]-F_{p^{i}}\left[x_{0}, y_{0}\right.\right.$, $\left.\left.\dot{y}_{0}, \lambda\left(x_{0}\right)\right]\right\}$. Then $Q(e) \geqq Q(0)=0$ for all sufficiently small $e, Q^{\prime}(0)=0$, and

$$
-\pi^{i} F_{p^{i} p^{k}}\left[x_{0}, y_{0}, \dot{y}_{0}, \lambda\left(x_{0}\right)\right] \dot{r}^{k}(0)=0 \text {. }
$$

Instead of (5.11) and (5.12) we infer from (5.14), (5.3), and (5.4) that $\psi_{p^{i}}^{\rho}\left(x_{0}, y_{0}, \dot{y}_{0}\right) \pi^{i}=\phi_{p^{i}}^{\delta}\left(x_{0}, y_{0}, \dot{y}_{0}\right) \pi^{i}=0$ for all $\rho$ and all $\delta$ in $\Delta\left(x_{0}\right)$, and this with (5.15) contradicts the nonsingularity hypothesis, since it is easy to see by differentiating the equations satisfied by $r(e)$ that $\dot{r}^{i}(0)=\pi^{i}$. If there is an index $\gamma$ in $\Gamma\left(x_{0}\right)-\Delta\left(x_{0}\right)$, we see from (5.13) and (5.14) that $\phi_{p^{i}}^{\gamma}\left(x_{0}, y_{0}, \dot{y}_{0}\right) \pi^{i} \geqq 0$. The modifications in the analysis for the bounded case can be easily carried out since this inequality holds.

To prove the converse of Theorem 5.1 we utilize the following lemma which is similar to the Corollary of Hestenes [3, Corollary 1, p. 59].

Lemma 5.5. There is a neighborhood $\mathcal{D}_{0}$ of $C_{0}$ relative to $\mathcal{D}$ and a positive constant $b_{1}$ such that

$$
E_{\phi \beta}(x, y, p, q)-\phi^{\beta}(x, y, q) \leqq b_{1} E_{L}(p, q)
$$


whenever $(x, y, p)$ is in $\mathcal{D}_{0}$ and $(x, y, q)$ is in $\mathcal{D}$. Moreover, if $H(x, y, p)$ is of class $C^{\prime \prime}$ on $R$ and is such that there exists a positive constant $W$ and a neighborhood $\mathcal{F}_{1}$ of $C_{0}$ in $(x, y)$-space such that $W L(p) \geqq|H(x, y, p)|$ whenever $(x, y)$ is in $\mathcal{F}_{1}$ and $(x, y, p)$ is in $\mathcal{D}$, then $\mathcal{D}_{0}$ and $b_{1}$ may be chosen so that

$$
\left|E_{H}(x, y, p, q)\right| \leqq b_{1} E_{L}(p, q)
$$

whenever $(x, y, p)$ is in $\mathcal{D}_{0}$ and $(x, y, q)$ is in $\mathcal{D}$.

Since $L_{p^{i} p^{k}} \pi^{i} \pi^{k}>0$ for all nonnull sets $\pi^{i}$, it follows from reasoning like that used in Lemma 5.3 that there is a neighborhood $R_{1}$ of $C_{0}$ in $(x, y, p)$-space and a positive constant $b_{2}$ such that (5.17) holds with $b_{1}$ replaced by $b_{2}$ whenever $(x, y, p)$ and $(x, y, q)$ are in $R_{1}$ for both $H$ and $\phi^{\beta}$. Since $\phi^{\beta} \geqq 0$ on $\mathcal{D}$, (5.16) also holds with $b_{1}$ replaced by $b_{2}$ whenever $(x, y, p)$ and $(x, y, q)$ are in $R_{1} D$. Choose a neighborhood $\mathcal{D}_{0}$ of $C_{0}$ relative to $\mathcal{D}$ such that the closure of $\mathcal{D}_{0}$ is in $R_{1}$. Then there exists a positive constant $b_{3}$ such that $L(q) \leqq b_{3} E_{L}(p, q)$ whenever $(x, y, p)$ is in $\mathcal{D}_{0}$ and $(x, y, q)$ is in $\mathcal{D}-\mathcal{R}_{1} \mathcal{D}$. Let $b_{4}$ be an upper bound for $\left|H(x, y, p)-p^{i} H_{p i}(x, y, p)\right|,\left|H_{p i}(x, y, p)\right|$, $\left|\phi^{\beta}(x, y, p)-p^{i} \phi_{p^{i}}^{\beta}(x, y, p)\right|,\left|\phi_{p^{i}}^{\boldsymbol{\beta}}(x, y, p)\right|$ whenever $(x, y, p)$ is in $\mathcal{D}_{0}$. If $(x, y, p)$ is in $\mathcal{D}_{0}$ and $(x, y, q)$ is in $\mathcal{D}-R_{1} \mathcal{D}$, then

$$
\left|E_{H}(x, y, p, q)-H(x, y, q)\right| \leqq b_{4}(n+1) L(q) \leqq b_{3} b_{4}(n+1) E_{L}(p, q),
$$

and the same inequality holds if $H$ is replaced by $\phi^{\beta}$. It follows that (5.16) is true if $b_{1}=b_{2}+b_{3} b_{4}(n+1)$ whenever $(x, y, p)$ is in $\mathcal{D}_{0}$ and $(x, y, q)$ is in $\mathcal{D}$. Reduce $\mathcal{D}_{0}$ if necessary so that $(x, y)$ is in $\mathcal{F}_{1}$ if $(x, y, p)$ is in $\mathscr{D}_{0}$. Then it is clear that (5.17) holds if $b_{1}=b_{2}+W b_{3}+b_{3} b_{4}(n+1)$ whenever $(x, y, p)$ is in $\mathcal{D}_{0}$ and $(x, y, q)$ is in $D$.

We may now complete the proof of Theorem 5.1. Suppose a positive constant $b$ and a neighborhood $R_{1}$ of $C_{0}$ in $(x, y, p)$ space exist such that the inequality (2.6) holds whenever $(x, y, p)$ is in $\mathcal{R}_{1} \mathcal{D},(x, y, q)$ is in $\mathcal{D}$, and $\phi^{\beta}(x, y, p)=0$ if $\beta$ is in $\Delta(x)$. By Lemma 5.5 we may find a positive constant $b_{1}$ and reduce $\mathcal{D}_{1}$ if necessary so that $\left|E_{\psi^{p}}\right| \leqq b_{1} E_{L},\left|E_{\phi}^{-\beta}\right| \leqq b_{1} E_{L}, E_{\phi^{\beta}}-\phi^{\beta}$ $\leqq b_{1} E_{L}$ if $(x, y, p)$ is in $R_{1} \mathcal{D}$ and $(x, y, q)$ is in $\mathcal{D}$. Define $N$ as the member of the class $\mathcal{N}$ determined by the neighborhood $R_{1}$ and a positive number $a$ such that $a<b, a b_{1}\left[t+1+m \max \left|\lambda^{\beta}(x)\right|\right]<b$. If $(x, y, p, q, \nu)$ is such that $(x, y, p, \nu)$ is in $N,(x, y, p)$ and $(x, y, q)$ are in $\mathcal{D}$, and $\phi^{\beta}(x, y, p)=0$ if $\beta$ is in $\Delta(x)$, then $\nu^{\gamma}\left(E_{\phi^{\gamma}}-\phi^{\gamma}\right) \geqq-a b_{1} E_{L}$ if $\gamma$ is in $\Gamma(x)-\Delta(x)$,

$$
\left(\nu^{\alpha}-\lambda^{\alpha}\right) E_{\bar{\phi}^{\alpha}} \geqq-a b_{1} \max \left|\lambda^{\alpha}(x)\right| E_{L}, \quad\left(\nu^{\alpha}-\lambda^{\alpha}\right) \phi^{\alpha} \geqq a \lambda^{\alpha}(x) \Phi^{\alpha},
$$

$\left(\nu^{p}-\lambda^{p}\right) E_{\nu^{p}} \geqq-a b_{1} E_{L}$. Now if $\alpha$ is in $\Delta(x)$, then $\phi^{\alpha}(x, y, p)=\Phi^{\alpha}(x, y, p)=0$, $\phi_{p}^{\alpha}(x, y, p)=\phi_{p^{i}}^{\alpha}(x, y, p)$, while if $\alpha$ is not in $\Gamma(x)$, then $\nu^{\alpha}=\lambda^{\alpha}=0$. Hence $\left(\nu^{\alpha}-\lambda^{\alpha}\right)\left(E_{\phi^{\alpha}}-\phi^{\alpha}\right)=\left(\nu^{\alpha}-\lambda^{\alpha}\right)\left(E_{\bar{\phi}^{\alpha}}-\bar{\phi}^{\alpha}\right)$ if $\alpha$ is restricted to the set complementary to $\Gamma(x)-\Delta(x)$. It follows from these relations and the identity (5.2), written in the form 


$$
\begin{aligned}
E_{F}(x, y, p, q, \nu) & -\nu^{\beta} \phi^{\beta}(x, y, q)=E_{F}(x, y, p, q, \lambda)-\lambda^{\beta}(x) \phi^{\beta}(x, y, q) \\
& +\left(\nu^{\rho}-\lambda^{\rho}\right) E_{\psi^{\rho}}+\nu^{\gamma}\left(E_{\phi}{ }^{\gamma}-\phi^{\gamma}\right)+\left(\nu^{\alpha}-\lambda^{\alpha}\right) E_{\bar{\phi}^{\alpha}}-\left(\nu^{\alpha}-\lambda^{\alpha}\right) \Phi^{\alpha},
\end{aligned}
$$

that the left-hand side of (5.1) is not less than

$$
\begin{aligned}
b\left[E_{L}(p, q)-\lambda^{\beta}(x) \Phi^{\beta}(x, y, q)\right]-a t b_{1} E_{L}(p, q)-a b_{1} E_{L}(p, q) \\
-a \max \left|\lambda^{\alpha}(x)\right| m b_{1} E_{L}(p, q)+a \lambda^{\alpha}(x) \Phi^{\alpha}(x, y, q) \geqq 0
\end{aligned}
$$

by virtue of our choice of $a$. This completes the proof of Theorem 5.1 since III' follows from Theorem 4.1.

6. Convergent sequences of admissible arcs. In the proof of Theorem 2.1 we shall need to be able to draw conclusions on the convergence of the derivatives $\dot{y}_{r}^{\boldsymbol{t}}$ of a sequence of admissible arcs $C_{r}$ which converge to $C_{0}$ uniformly in $(x, y)$-space. The particular results needed can be deduced from the following theorem.

TheOREM 6.1. Let $C_{0}$ satisfy the hypotheses of Theorem 2.1. If $C_{r}$ is a sequence of admissible arcs in $\mathcal{D}$ which converge uniformly to $C_{0}$ in $(x, y)$-space such that $\lim \sup J\left(C_{r}\right) \leqq J\left(C_{0}\right)$, then it is true that $\lim K\left(C_{r}, C_{0}\right)=0$, and that there is a subsequence $C_{r_{k}}$ of the sequence $C_{r}$ such that $\lim \dot{y}_{r_{k}}^{\prime}=\dot{y}_{0}^{\prime}$ almost uniformly on $x^{1} x^{2}$.

Here $J(C)$ and $K\left(C, C_{0}\right)$ are defined as

$$
\begin{gathered}
J(C)=\int_{x^{1}}^{x^{2}}\left[F(x, y, \dot{y}, \lambda)-\lambda^{\beta}(x) \phi^{\beta}(x, y, \dot{y})\right] d x, \\
K\left(C, C_{0}\right)=\int_{x_{1}}^{x^{2}}\left[L\left(\dot{y}-\dot{y}_{0}\right)-1\right] d x .
\end{gathered}
$$

Consider the equations

$$
\begin{gathered}
\phi^{\beta}(x, y, P)=\phi^{\beta}\left(x, y_{0}, \dot{y}_{0}\right), \quad \psi^{\rho}(x, y, P)=0, \\
\psi^{i}(x, y, P)=\psi^{i}\left(x, y_{0}, \dot{y}_{0}\right)
\end{gathered}
$$

in which the functions $\psi^{j}$ are those of Lemma 4.1. By Corollary 2 to Theorem 4.1 the functions involved in equations (6.1) are of class $C^{\prime}$ for $x$ on each $\bar{A}_{\tau}$ and for $(x, y, P)$ near $C_{0}$. Hence there exist solutions $P^{i}(x, y)$ of class $C^{\prime}$ if $x$ is in $\bar{A}_{\tau}$ and $y$ is near $y_{0}(x)$ such that $P^{i}\left[x, y_{0}(x)\right]=\dot{y}_{0}^{i}(x)$. Since $\dot{y}_{0}^{i}(x)$ is continuous, it follows that equations (6.1) are satisfied when $x$ is an end point $x_{\tau}$ of an interval $A_{\tau}$ by $P^{i}\left(x_{\tau}^{-}, y\right)$ as well as by $P^{i}\left(x_{\tau}+, y\right)$. Since the equations (6.1) admit only one solution near $\dot{y}_{0}^{4}(x)$ when $y^{4}$ is sufficiently near $y_{0}^{8}(x)$, it follows that $P^{i}\left(x_{\tau}^{-}, y\right)=P^{i}\left(x_{\tau}^{+}, y\right)$, and hence that $P^{i}(x, y)$ is continuous on the whole interval $x^{1} x^{2}$. Differentiating this last equation we see also that $P_{y^{k}}^{t}(x, y)$ is continuous on $x^{1} x^{2}$.

With the help of the functions $P^{i}(x, y), J(C)$ can be written as the sum 


$$
J(C)=J^{*}(C)+E^{*}(C),
$$

in which

$$
\begin{aligned}
& J^{*}(C)=\int_{x^{1}}^{x^{2}}\left[F(x, y, P, \lambda)+\left(\dot{y}^{i}-P^{i}\right) F_{p^{i}}(x, y, P, \lambda)\right] d x, \\
& E^{*}(C)=\int_{x^{1}}^{x^{2}}\left[E_{F}(x, y, P, \dot{y}, \lambda)-\lambda^{\beta}(x) \phi^{\beta}(x, y, \dot{y})\right] d x .
\end{aligned}
$$

For any function $H(x, y, p)$ of class $C^{\prime \prime}$ on $\mathcal{D}$ we define

$$
H(C)=\int_{x^{1}}^{x^{2}} H(x, y, \dot{y}) d x .
$$

We shall be interested only in the case in which there is a positive constant $c$ and a neighborhood $\mathscr{D}_{0}$ of $C_{0}$ relative to $\mathscr{D}$ such that

$$
E_{F}(x, y, p, q, \lambda)-\lambda^{\beta}(x) \phi^{\beta}(x, y, q) \geqq c\left|E_{H}(x, y, p, q)\right|
$$

whenever $(x, y, p)$ is in $\mathcal{D}_{0},(x, y, q)$ is in $\mathcal{D}$, and $\phi^{\beta}(x, y, p)=0$ if $\beta$ is in $\Delta(x)$. We shall say that $H$ is $E^{*}$-dominated by $F$ near $C_{0}$ on $\mathcal{D}$ when this is true.

We shall also restrict the constant $c$ and the neighborhood $D_{0}$ so that

$$
E_{F}(x, y, p, q, \lambda)-\lambda^{\beta}(x) \phi^{\beta}(x, y, q)=c E_{L}(p-P, q-P)
$$

whenever $(x, y, p)$ is in $\mathscr{D}_{0},(x, y, q)$ is in $\mathcal{D}$, and $\phi^{\beta}(x, y, p)=0$ if $\beta$ is in $\Delta(x)$. The possibility of doing this follows from the $E^{*}$-dominance of $L$ by $F$ near $C_{0}$ on $\mathcal{D}$ and from Lemma 5.5 when we observe that $L(p-P)$ satisfies the hypotheses imposed on $H(x, y, p)$ in that Lemma.

Theorem 6.1 will be a consequence of the following lemma, whose proof is identical with that of a similar lemma of Hestenes [4, Theorem 5.1].

Lemma 6.1. Let $C_{0}$ satisfy the hypotheses of Theorem 2.1. Given a constant $\epsilon>0$, there exists a constant $\eta>0$ and a neighborhood $\mathcal{F}$ of $C_{0}$ in $(x, y)$-space such that the inequality

$$
H(C)-H\left(C_{0}\right)<\epsilon
$$

holds for every admissible arc $C$ in $\mathcal{F}$ which lies in $\mathcal{D}$, satisfies the end conditions (2.1), and is such that

$$
J(C) \leqq J\left(C_{0}\right)+\eta .
$$

The first part of Theorem 6.1 will follow from Lemma 6.1 provided that $H(C)=K\left(C, C_{0}\right)$ has an integrand $H(x, y, p)=L\left(p-\dot{y}_{0}\right)-1$ which is $E^{*}$-dominated by $F$ near $C_{0}$ on $\mathscr{D}$. Since this can be shown just as (6.5) was shown, we conclude that

$$
\lim K\left(C_{r}, C_{0}\right)=0
$$


To prove the second part of the theorem, we observe that Schwarz' inequality implies that

$$
\begin{aligned}
\left\{\int_{x^{1}}^{x^{2}}\left|\dot{y}_{r}-\dot{y}_{0}\right| d x\right\}^{2} & =\left\{\int_{x^{1}}^{x^{2}}\left[L\left(\dot{y}_{r}-\dot{y}_{0}\right)-1\right]^{1 / 2}\left[L\left(\dot{y}_{r}-\dot{y}_{0}\right)+1\right]^{1 / 2} d x\right\}^{2} \\
& \leqq K\left(C_{r}, C_{0}\right) \int_{x^{1}}^{x^{2}}\left[L\left(\dot{y}_{r}-\dot{y}_{0}\right)+1\right] d x \\
& =K\left(C_{r}, C_{0}\right)\left[2\left(x^{2}-x^{1}\right)+K\left(C_{r}, C_{0}\right)\right] .
\end{aligned}
$$

It follows that $\dot{y}_{\tau}^{i}$ converges in mean of order one to $\dot{y}_{0}^{t}$. The existence of a subsequence which converges almost everywhere to $\dot{y}_{0}^{4}$ (and hence almost uniformly to $\dot{y}_{0}^{q}$ ) is a well known consequence of convergence in mean [2, Theorem 23, p. 242 and Theorem 19, p. 239].

7. The variation $\eta_{0}$. Our proof of Theorem 2.1 is indirect. Suppose the hypotheses of Theorem 2.1 are fulfilled but that the conclusion is not. Then there is a sequence $C_{r}: y^{i}=y_{r}^{i}(x)$ of curves in $\mathcal{D}$ which satisfy the end conditions (2.1), are different from $C_{0}$, and such that

$$
\lim y_{r}^{i}(x)=y_{0}^{i}(x)
$$

uniformly on $x^{1} x^{2}$, and yet $I\left(C_{r}\right) \leqq I\left(C_{0}\right)$. Since $C_{r}$ and $C_{0}$ are in $\Phi$ and $\lambda^{0} \geqq 0, J\left(C_{r}\right)=\lambda^{0} I\left(C_{r}\right) \leqq \lambda^{0} I\left(C_{0}\right)=J\left(C_{0}\right)$. By virtue of Theorem 6.1 we may replace $C_{r}$ by a subsequence for which $\dot{y}_{r}^{t}$ converges almost uniformly to $\dot{y}_{0}^{4}$ on $x^{1} x^{2}$ and hence for which $\lim k_{r}=0$, in which

$$
k_{r} \geqq 0, \quad k_{r}^{2}=K\left(C_{r}, C_{0}\right)-\int_{x^{1}}^{x^{2}} \lambda^{\beta}(x) \phi^{\beta}\left(x, y_{r}, \dot{y}_{r}\right) d x .
$$

This follows since $\lambda^{\beta}(x) \Phi^{\beta}\left(x, y_{r}, \dot{y}_{r}\right)$ converges boundedly to $\lambda^{\beta}(x) \Phi^{\beta}\left(x, y_{0}, \dot{y}_{0}\right)$ $=0$ almost everywhere on $x^{1} x^{2}$. Let us define

$$
\eta_{r}^{i}(x)=\left(y_{r}^{i}-y_{0}^{i}\right) / k_{r} .
$$

Then it is clear that

$$
\int_{x^{1}}^{x^{2}}\left\{\left|\dot{\eta}_{r}\right|^{2} / h_{r}(x)\right\} d x \leqq 1,
$$

in which

$$
h_{r}(x)=1+L\left(\dot{y}_{r}-\dot{y}_{0}\right)=k_{r}^{2}\left|\dot{\eta}_{r}\right|^{2} /\left[L\left(\dot{y}_{r}-\dot{y}_{0}\right)-1\right] .
$$

LEMMA 7.1. The integrals of the functions $h_{r}(x)$ are absolutely continuous uniformly with respect to $r$.

This follows since $h_{r}(x)$ differs by 2 from the integrand of $K\left(C_{r}, C_{0}\right)$ and $K\left(C_{r}, C_{0}\right)$ tends to zero. 
LEMMA 7.2. The functions $\eta_{r}^{\$}(x)$ are absolutely continuous uniformly with respect to $r$.

By Schwartz's inequality and (7.1),

$$
\left|\int_{M} \dot{\eta}_{r}^{i} d x\right|^{2} \leqq \int_{M}\left(\left|\dot{\eta}_{r}\right|^{2} / h_{r}\right) d x \int_{M} h_{r} d x \leqq \int_{M} h_{r} d x
$$

Thus the result follows from Lemma 7.1.

LEMMA 7.3. The sequence of arcs $C_{r}$ may be chosen so that there exists a function $\eta_{0}^{i}(x)$ satisfying the end conditions (2.8) and such that $\lim \eta_{r}^{i}(x)=\eta_{0}^{i}(x)$ uniformly on $x^{1} x^{2}$. Moreover, $\eta_{0}^{4}(x)$ is absolutely continuous and

$$
\int_{x^{1}}^{x^{2}}\left|\dot{\eta}_{0}\right|^{2} d x \leqq 2 .
$$

By Lemma 7.2 the functions $\eta_{r}^{\prime}(x)$ are absolutely continuous uniformly in $r$. Since $\eta_{r}^{i}\left(x^{1}\right)=0$, the functions $\eta_{r}^{\imath}(x)$ are also uniformly bounded. By Ascoli's theorem [2, p. 122] subsequences can be found which converge uniformly to limits $\eta_{0}^{3}(x)$ and these limits are obviously absolutely continuous and such that $\eta_{0}^{s}\left(x^{s}\right)=0$.

The proof of the integrability of $\left|\dot{\eta}_{0}\right|^{2}$ to an integral bounded by two is identical with that of the corresponding assertion when there are no differential inequalities [6, pp. 528-529].

LEMмA 7.4. If $g(x)$ is bounded and measurable, and if $N_{i r}(x)$ are continuous functions which converge uniformly to $N_{i 0}(x)$ on $x^{1} x^{2}$, then

$$
\begin{aligned}
& \lim \int_{M} g(x)\left(\eta_{r}^{i}-\eta_{0}^{i}\right) d x=0 \\
& \lim \int_{M} N_{i r}(x) \dot{\eta}_{r}^{i} d x=\int_{M} N_{i 0}(x) \dot{\eta}_{0}^{i} d x
\end{aligned}
$$

for every measurable subset $M$ of $x^{1} x^{2}$. If $|g(x)|^{2}$ is integrable, then

$$
\lim \int_{M} g(x)\left(\dot{\eta}_{r}^{i}-\dot{\eta}_{0}^{i}\right) d x=0
$$

for every measurable subset $M$ of $x^{1} x^{2}$ on which $\dot{y}_{r}^{\prime}$ converges uniformly.

The relation (7.3) is obvious since $\eta_{r}^{i}(x)$ converges uniformly to $\eta_{0}^{4}(x)$. If $|g(x)|^{2}$ is integrable, there exists for each $\epsilon>0$ a bounded function $G(x)$ such that $[8$, p. 229]

$$
\int_{x^{1}}^{x^{2}}|g(x)-G(x)|^{2} d x<\epsilon .
$$


If $M$ is a set on which $\dot{y}_{r}^{t}$ converges uniformly to $\dot{y}_{0}^{t}$ on $M$, then $h_{r}(x)$ converges uniformly to 2 on $M$ and so it follows from (7.1) and Lemma 7.3 that

$$
\int_{M}\left|\dot{\eta}_{r}\right|^{2} d x \leqq 3, \quad \int_{M}\left|\dot{\eta}_{0}\right|^{2} d x \leqq 2
$$

if $r$ is sufficiently large. From Schwarz' inequality we then have

$$
\begin{aligned}
\left|\int_{M}[g(x)-G(x)]\left(\dot{\eta}_{r}^{i}-\dot{\eta}_{0}^{i}\right) d x\right|^{2} \\
\qquad 2 \int_{M}[g(x)-G(x)]^{2} d x \int_{M}\left(\left|\dot{\eta}_{r}\right|^{2}+\left|\dot{\eta}_{0}\right|^{2}\right) d x \leqq 10 \epsilon .
\end{aligned}
$$

To prove (7.5) it is therefore sufficient to prove it when $g(x)$ is bounded. The relation (7.5) when $g(x)$ is bounded and $M$ is any measurable subset of $x^{1} x^{2}$ and the relation (7.4) follow from known results on Lebesgue-Stieltjes integrals [see 2, Theorem 28, p. 285; Theorem 21, p. 280; Corollary to Theorem 20, p. 280].

8. Some auxiliary functions. Let us recall from $\$ 6$ the definition of the functions $P^{i}(x, y)$ such that $[x, y, P(x, y)]$ lies in $\mathcal{D}$ whenever $(x, y)$ is in a neighborhood of $C_{0}$ and such that $P^{i}\left(x, y_{0}\right)=\dot{y}_{0}^{t}$. Define $p_{r}^{i}(x)=P^{i}\left[x, y_{r}(x)\right]$, $\pi_{r}^{i}(x)=\left[p_{r}^{i}(x)-p_{0}^{i}(x)\right] / k_{r}, \pi_{0}^{i}(x)=P_{y}^{i}\left[x, y_{0}(x)\right] \eta_{0}^{0}(x)$.

LEMMA 8.1. The following relations hold:

$$
\begin{aligned}
\phi^{\beta}\left(x, y_{r}, p_{r}\right) & =\phi^{\beta}\left(x, y_{0}, \dot{y}_{0}\right), \quad \psi^{\rho}\left(x, y_{r}, p_{r}\right)=0, \\
\lim p_{r}^{i}(x) & =p_{0}^{i}(x)=\dot{y}_{0}^{i}(x) \text { uniformly on } x^{1} x^{2}, \\
\lim \pi_{r}^{i}(x) & =\pi_{0}^{i}(x) \text { uniformly on } x^{1} x^{2} .
\end{aligned}
$$

Equations (8.1) are immediate consequences of the definitions. Equations (8.2) follow from the uniform convergence of $y_{r}^{4}(x)$ to $y_{0}^{4}(x)$ and the continuity of $P^{i}(x, y)$ for $x$ on $x^{1} x^{2}$ and $y$ near $y_{0}(x)$. To prove equations (8.3) observe that Taylor's formula yields

$$
\pi_{r}^{i}(x)=B_{v r}^{i}(x) \eta_{r}^{v}(x)
$$

in which

$$
B_{v r}^{i}(x)=\int_{0}^{1} P_{y^{v}}^{i}\left[x, y_{0}+\theta\left(y_{r}-y_{0}\right)\right] d \theta .
$$

Since $y_{r}^{4}$ converges uniformly to $y_{0}^{4}$ and since $P_{p^{v}}^{i}(x, y)$ is continuous for $x$ on $x^{1} x^{2}$ and $y$ near $y_{0}(x), B_{v r}^{i}(x)$ converges uniformly to $P_{\nu^{j}}^{i}\left[x, y_{0}(x)\right]$. By Lemma 7.3, $\eta_{r}^{0}(x)$ converges uniformly to $\eta_{0}^{0}(x)$. Hence equations $(8.3)$ are true.

As a corollary of Lemmas 8.1 and 7.4 we have the following lemma. 
LEMMA 8.2. If $N_{i r}(x)$ are continuous functions which converge uniformly to $N_{i 0}(x)$ on $x^{1} x^{2}$, and if $|g(x)|^{2}$ is integrable, then

$$
\lim \int_{M} N_{i r}\left(\dot{\eta}_{r}^{i}-\pi_{r}^{i}\right) d x=\int_{M} N_{i 0}\left(\dot{\eta}_{0}^{i}-\pi_{0}^{i}\right) d x
$$

for every measurable subset $M$ of $x^{1} x^{2}$, and

$$
\lim \int_{M} g(x)\left(\dot{\eta}_{r}^{i}-\pi_{r}^{i}\right) d x=\int_{M} g(x)\left(\dot{\eta}_{0}^{i}-\pi_{0}^{i}\right) d x
$$

for every measurable subset $M$ of $x^{1} x^{2}$ on which $\dot{y}_{r}^{4}$ converges uniformly.

LEMMA 8.3. If $\phi(x, y, p)$ is any function of class $C^{\prime}$ near $C_{0}$, then

$$
\lim k_{r}^{-1}\left[\phi\left(x, y_{r}, p_{r}\right)-\phi\left(x, y_{0}, \dot{y}_{0}\right)\right]=\phi_{y_{i}} \eta_{0}^{i}+\phi_{p} i \pi_{0}^{i}
$$

uniformly on $x^{1} x^{2}$.

This follows directly from Taylor's theorem and Lemma 8.1.

If we replace, in Lemma $8.3, \phi$ by $\psi^{\rho}$ and then by $\phi^{\beta}$ and use equations (8.1) we immediately deduce the following lemma.

LEMMA 8.4. The functions $\eta_{0}^{i}(x)$ satisfy with the auxiliary functions $\pi_{0}^{s}(x)$ the following equations:

$$
\psi_{y i \eta_{0}^{p}}^{i}+\psi_{p^{i} \pi_{0}^{i}}^{i}=0, \quad \phi_{y^{i} \eta_{0}^{i}}^{i}+\phi_{p^{i}}^{\beta} \pi_{0}^{i}=0 .
$$

9. First order terms. Let $H(x, y, p)$ be a function of class $C^{\prime}$ near $C_{0}$, and define

$$
\begin{aligned}
H(C, M) & =\int_{M} H(x, y, \dot{y}) d x, \\
H^{*}(C, M) & =\int_{M}\left[H(x, y, P)+\left(\dot{y}^{i}-P^{i}\right) H_{p^{i}}(x, y, P)\right] d x, \\
E_{H}^{*}(C, M) & =\int_{M} E_{H}(x, y, P, \dot{y}) d x, \\
H_{1}(\eta, M) & =\int_{M}\left(H_{y} \eta^{i}+H_{p \cdot \dot{\eta}^{i}}\right) d x .
\end{aligned}
$$

It is clear that

$$
H(C, M)=H^{*}(C, M)+E_{H}^{*}(C, M) .
$$

Lemma 9.1. If $H(x, y, p)$ is of class $C^{\prime}$ near $C_{0}$, then

$$
\lim k_{r}^{-1}\left[H^{*}\left(C_{r}, M\right)-H^{*}\left(C_{0}, M\right)\right]=H_{1}\left(\eta_{0}, M\right) .
$$


This follows at once from Lemmas 8.3 and 8.2.

Let us define

$$
J_{1}(\eta)=\int_{x^{1}}^{x^{2}}\left(F_{y} \eta^{i}+F_{p i} \dot{\eta}^{i}\right) d x .
$$

By virtue of equation (2.3) and the fact that $\eta_{r}^{\sharp}\left(x^{s}\right)=0$, we have the following lemma.

LEMMA 9.2. For each $r=0,1, \cdots, J_{1}\left(\eta_{r}\right)=\left.F_{p} \eta_{r}^{i}\right|_{x^{1}} ^{x^{2}}=0$.

LEMma 9.3. We have that

$\lim k_{r}^{-1}\left[J\left(C_{r}\right)-J\left(C_{0}\right)\right]=\lim k_{r}^{-1}\left[J^{*}\left(C_{r}\right)-J^{*}\left(C_{0}\right)\right]=\lim k_{r}^{-1} E^{*}\left(C_{r}\right)=0$.

By Lemmas 9.1 and 9.2 we have that

$$
\lim k_{r}^{-1}\left[J^{*}\left(C_{r}\right)-J^{*}\left(C_{0}\right)\right]=J_{1}\left(\eta_{0}\right)=0 .
$$

From equation (6.7) we conclude that

$$
0 \geqq \lim \sup k_{r}^{-1}\left[J\left(C_{r}\right)-J\left(C_{0}\right)\right]=\lim \sup k_{r}^{-1} E^{*}\left(C_{r}\right) .
$$

However, $E^{*}\left(C_{r}\right) \geqq 0$ if $r$ is so large that $\left(x, y_{r}, p_{r}\right)$ is near enough to $C_{0}$ for (2.6) to hold, since both $\left(x, y_{r}, p_{r}\right)$ and $\left(x, y_{r}, \dot{y}_{r}\right)$ are in $\mathcal{D}$ and $\phi^{\beta}\left(x, y_{r}, p_{r}\right)=0$ if $\beta$ is in $\Delta(x)$. Hence the right-hand side of (9.2) cannot be negative and so the lemma is true.

Lemma 9.4. If $H(x, y, p)$ is of class $C^{\prime}$ near $C_{0}$ and is $E^{*}$-dominated by $F$ near $C_{0}$ on $\mathcal{D}$, then

$$
\lim k_{r}^{-1}\left[H\left(C_{r}, M\right)-H\left(C_{0}, M\right)\right]=H_{1}\left(\eta_{0}, M\right) .
$$

If $r$ is large enough, we may integrate the inequality (6.4) to see that $\left|E_{H}^{*}\left(C_{r}, M\right)\right| \leqq C^{-1} E^{*}\left(C_{r}\right)$ and so we see from Lemma 9.3 that

$$
\lim k_{r}^{-1} E_{H}^{*}\left(C_{r}, M\right)=0 .
$$

Lemma 9.4 is now an immediate consequence of Lemma 9.1 and equation (9.1).

10. Admissibility of the variation $\eta_{0}^{i}$. We have seen in Lemma 7.3 that the functions $\eta_{0}^{3}$ are absolutely continuous, have integrable square derivatives, and satisfy the end conditions (2.8). We complete the proof of the admissibility of $\eta_{0}^{\sharp}$ in the following lemma.

Lemma 10.1. The variation $\eta_{0}^{\prime}$ satisfies (2.9) for almost all $x$ in $B(\beta),(2.10)$ for almost all $x$ in $A(\beta)$, and (2.11) for almost all $x$ on $x^{1} x^{2}$.

It follows from Lemma 5.5 that the functions $\psi^{\rho}$ and $\phi^{\beta}$ satisfy the conditions imposed on $H$ in Lemma 9.4. We thus infer that 


$$
0=\lim k_{r}^{-1} \int_{M}\left[\psi^{\rho}\left(x, y_{r}, \dot{y}_{r}\right)-\psi^{\rho}\left(x, y_{0}, \dot{y}_{0}\right)\right] d x=\int_{M}\left(\psi_{y^{i} \eta_{0}}^{i}+\psi_{p}^{\rho} \dot{\eta_{0}}\right) d x
$$

for every measurable subset $M$ of $x^{1} x^{2}$. Hence (2.11) is satisfied for almost all $x$ on $x^{1} x^{2}$. We also have that

$$
\begin{gathered}
0 \leqq \lim k_{r}^{-1} \int_{M} \phi^{\beta}\left(x, y_{r}, \dot{y}_{r}\right) d x=\int_{M}\left(\phi_{y^{\prime} \eta_{0}}^{i}+\phi_{p i}^{\beta} \dot{\eta}_{0}^{i}\right) d x, \\
0 \leqq \int_{M}\left(\begin{array}{c}
\beta \\
\phi_{y}^{*} \eta_{0}^{i}
\end{array}+\phi_{p i \dot{\eta}_{0}}^{\beta}\right) d x
\end{gathered}
$$

for every measurable subset $M$ of $A(\beta)$. Hence (2.10) is satisfied for almost all $x$ in $A(\beta)$. If $M$ is a closed subset of $B(\beta)$, there is a number $\epsilon_{\beta}(M)$ such that $\lambda^{\beta}(x) \leqq-\epsilon_{\beta}(M)<0$ on $M$. Hence it follows from the definition of $k_{r}$ that

$$
\begin{aligned}
0 \leqq k_{r}^{-1} \int_{M} \phi^{\beta}\left(x, y_{r}, \dot{y}_{r}\right) d x & \leqq-k_{r}^{-1} \epsilon_{\beta}^{-1}(M) \int_{x 1}^{x^{2}} \lambda^{\beta}(x) \phi^{\beta}\left(x, x_{r}, \dot{y}_{r}\right) d x \\
& \leqq k_{r} \epsilon_{\beta}^{-1}(M) .
\end{aligned}
$$

Hence the inequality (10.1) is an equality for every closed subset $M$ of $B(\beta)$. It follows that (2.9) is satisfied for almost all $x$ in $B(\beta)$.

11. Second order terms. The second variation of $J^{*}(C)$ along $C_{0}$ is

$$
J_{2}^{*}(\eta)=\int_{x 1}^{x^{2}}\left[2 \omega\left(x, \eta, \pi_{0}\right)+2\left(\dot{\eta}^{i}-\pi_{0}^{i}\right) \omega_{\pi_{i}}\left(x, \eta, \pi_{0}\right)\right] d x
$$

in which $2 \omega$ is defined in (2.12). It is easy to see that

$$
J_{2}^{*}(\eta)=J_{2}(\eta)-\int_{x^{1}}^{x^{2}} F_{p^{i} p^{v}}\left(\dot{\eta}^{i}-\pi_{0}^{i}\right)\left(\dot{\eta}^{v}-\pi_{0}^{v}\right) d x .
$$

Lemma 11.1. We have that $\lim k_{r}^{-2}\left[J^{*}\left(C_{r}\right)-J^{*}\left(C_{0}\right)\right]=(1 / 2) J_{2}^{*}\left(\eta_{0}\right)$.

This follows at once from Taylor's theorem and Lemmas 9.2 and 8.2.

LemMa 11.2. If $H(x, y, p)$ is a function of the form

$$
H=\theta\left[\psi^{\rho}(x, y, p) \psi^{\rho}(x, y, p)+\phi_{0}^{\beta}(x, y, p) \phi_{0}^{\beta}(x, y, p)\right],
$$

in which $\theta$ is constant and $\phi_{0}^{\beta}=\lambda^{\beta}(x) \phi^{\beta}(x, y, p)$ ( $\beta$ not summed $)$, then

$$
\lim k_{r}^{-2} \int_{M} E_{H}\left(x, y_{r}, p_{r}, \dot{y}_{r}\right) d x=0
$$

for every measurable subset $M$ of $x^{1} x^{2}$ on which $\dot{y}_{r}^{3}$ converges uniformly.

Since equations (8.1) hold, the lemma is equivalent to proving that 


$$
\lim k_{r}^{-2} \int_{M} \phi_{0}^{\beta}\left(x, y_{r}, \dot{y}_{r}\right) \phi_{0}^{\beta}\left(x, y_{r}, \dot{y}_{r}\right) d x=0
$$

for each $\beta$. Since $\dot{y}_{r}^{i}$ converges uniformly to $\dot{y}_{0}^{i}$ on $M$ and (2.4) holds, there exists for each $\epsilon>0$ an index $R(\epsilon)$ such that

$$
0 \geqq \phi_{0}^{\beta}\left(x, y_{r}, \dot{y}_{r}\right)\left[1+\phi^{\beta^{2}}\left(x, y_{r}, \dot{y}_{r}\right)\right]^{1 / 2} \geqq-\epsilon
$$

for each $\beta$ if $r>R(\epsilon)$. By the definition of $k_{r}$, we have that if $r>R(\epsilon)$,

$$
0 \leqq k_{r}^{-2} \int_{M} \phi_{0}^{\beta}\left(x, y_{r}, \dot{y}_{r}\right) \phi_{0}^{\beta}\left(x, y_{r}, \dot{y}_{r}\right) d x \leqq-k_{r}^{-2} \epsilon \int_{M} \lambda^{\beta}(x) \phi^{\beta}\left(x, y_{r}, \dot{y}_{r}\right) d x \leqq \epsilon
$$

for each $\beta$. Hence the lemma is true.

\section{LEMma 11.3. We have that}

$$
\lim \inf k_{r}^{-2} E^{*}\left(C_{r}\right) \geqq \frac{1}{2} \int_{x_{1}}^{x^{2}} F_{p^{i} p^{v}}\left(\dot{\eta}_{0}^{i}-\pi_{0}^{i}\right)\left(\dot{\eta}_{0}^{v}-\pi_{0}^{v}\right) d x
$$

In order to prove this result let $M$ be a measurable subset of $x^{1} x^{2}$ on which $\dot{y}_{r}^{i}$ converges uniformly to $\dot{y}_{0}^{i}$. With the help of Lemma 11.2 and Corollary 3 to Theorem 4.1 the proof of the relation

$$
\lim \inf {k_{r}^{-2}}^{-2} E_{M}\left(x, y_{r}, p_{r}, \dot{y}_{r}, \lambda\right) d x \geqq \frac{1}{2} \int_{M} F_{p^{i} p^{v}}\left(\dot{\eta}_{0}^{i}-\pi_{0}^{i}\right)\left(\dot{\eta}_{0}^{v}-\pi_{0}^{v}\right) d x
$$

can be made by the method of Hestenes [5, Lemma 10.1]. Since $\lambda^{\beta}(x) \phi^{\beta}\left(x, y_{r}, \dot{y}_{r}\right) \leqq 0$ and since (2.6) holds, we thus find that

$\lim \inf k_{r}^{-2} E^{*}\left(C_{r}\right) \geqq \lim \inf {k_{r}}^{-2} \int_{M}\left[E_{F}\left(x, y_{r}, p_{r}, \dot{y}_{r}, \lambda\right)-\lambda^{\beta}(x) \phi^{\beta}\left(x, y_{r}, \dot{y}_{r}\right)\right] d x$

$$
\begin{aligned}
& \geqq \lim \inf k_{r}^{-2} \int_{M} E_{F}\left(x, y_{r}, p_{r}, \dot{y}_{r}, \lambda\right) d x \\
& \geqq \frac{1}{2} \int_{M} F_{p^{i} p^{v}}\left(\dot{\eta}_{0}^{i}-\pi_{0}^{v}\right)\left(\dot{\eta}_{0}^{v}-\pi_{0}^{v}\right) d x .
\end{aligned}
$$

Since $\dot{y}_{r}^{i}$ converges almost uniformly on $x^{1} x^{2}$, it follows from our choice of $M$ and the integrability of $\left|\dot{\eta}_{0}\right|^{2}$ that this last inequality also holds when $M$ is replaced by the whole interval $x^{1} x^{2}$. Hence the lemma is true.

12. Completion of the proof of Theorem 2.1. By virtue of the definition of $C_{r}$ and equation (6.7), we have that

$$
0 \geqq k_{r}^{-2}\left[J\left(C_{r}\right)-J\left(C_{0}\right)\right]=k_{r}^{-2}\left[J^{*}\left(C_{r}\right)-J^{*}\left(C_{0}\right)+E^{*}\left(C_{r}\right)\right] .
$$

By Lemmas 11.1, 11.3 and equation above Lemma 11.1, we have that 
$0 \geqq J_{2}\left(\eta_{0}\right)$. Since $\eta_{0}^{i}$ is an admissible variation by Lemmas 7.3 and 10.1 , it follows from the hypothesis of Theorem 2.1 that $\eta_{0}^{i}(x)=0$. It then follows from (12.1) and the non-negativeness of $E^{*}\left(C_{r}\right)$ for sufficiently large $r$ that $\lim k_{r}^{-2} E^{*}\left(C_{r}\right)=0$. By Lemma 5.5 there exists a positive number $b^{*}$, which we may assume to be less than one, such that $E_{L}\left(p_{r}, \dot{y}_{r}\right) \geqq b^{*} E_{L}\left(p_{r}-\dot{y}_{0}, \dot{y}_{r}-\dot{y}_{0}\right)$ for $r$ sufficiently large. It then follows from (2.6) that for sufficiently large $r$,

$$
\begin{aligned}
k_{r}^{-2} E^{*}\left(C_{r}\right) & \geqq b b^{*} k_{r}^{-2} \int_{x^{1}}^{x^{2}}\left[E_{L}\left(p_{r}-\dot{y}_{0}, \dot{y}_{r}-\dot{y}_{0}\right)-\lambda^{\beta}(x) \Phi^{\beta}\left(x, y_{r}, \dot{y}_{r}\right)\right] d x \\
& \geqq b b^{*} k_{r}^{-2} \int_{x^{1}}^{x^{2}}\left[L\left(\dot{y}_{r}-\dot{y}_{0}\right)-\frac{1+k_{r}^{2} \dot{\eta}_{r}^{i} \pi_{r}^{i}}{L\left(p_{r}-\dot{y}_{0}\right)}-\lambda^{\beta}(x) \Phi^{\beta}\left(x, y_{r}, \dot{y}_{r}\right)\right] d x \\
& \geqq b b^{*} k_{r}^{-2} \int_{x^{1}}^{x^{2}}\left[L\left(\dot{y}_{r}-\dot{y}_{0}\right)-1-\lambda^{\beta}(x) \Phi^{\beta}\left(x, y_{r}, \dot{y}_{r}\right)-\frac{k_{r}^{2} \dot{\eta}_{r}^{i} \pi_{r}^{i}}{L\left(p_{r}-\dot{y}_{0}\right)}\right] d x .
\end{aligned}
$$

By Lemmas 7.4 and 8.1 and the definition of $k_{r}$ we find that

$$
\lim \inf k_{r}^{-2} E^{*}\left(C_{r}\right) \geqq b b^{*}>0
$$

since $\eta_{0}^{4} \equiv 0$, and this is a contradiction from which we infer the truth of Theorem 2.1.

\section{BIBLIOGRAPHY}

1. Gilhert A. Bliss, Lectures in the calculus of variations, University of Chicago Press, 1946.

2. Lawrence M. Graves, Theory of functions of real variables, McGraw-Hill, 1946.

3. M. R. Hestenes, The Weierstrass E-function in the calculus of variations, Trans. Amer. Math. Soc. vol. 60 (1946) pp. 51-71.

4. —, Theorem of Lindberg in the calculus of variations, ibid. pp. 72-92.

5. - Sufficient conditions for the isoperimetric problem of Bolza in the calculus of variations, ibid. pp. 93-118.

6. - An indirect sufficiency proof for the problem of Bolza in nonparametric form, ibid. vol. 62 (1947) pp. 509-535.

7. William Karush, Minima of functions of several variables with inequalities as side conditions, University of Chicago Master's Thesis, 1939.

8. E. J. McShane, Integration, Princeton University Press, 1944.

9. - Sufficient conditions for a weak relative minimum in the problem of Bolza, Trans. Amer. Math. Soc. vol. 52 (1942) pp. 344-379.

10. W. T. Reid, Isoperimetric problems of Bolza in nonparametric form, Duke Math. J. vol. 5 (1939) pp. 675-691.

11. Frederick A. Valentine, The problem of Lagrange with differential inequalities as added side conditions, Contributions to the Calculus of Variations 1933-37, University of Chicago Press.

UNIVERSITY OF ILLINOIS,

Chicago, Ill. 